\title{
A Study on Verification of Equivalence and Effectiveness of Non-Pharmacologic Dementia Prevention and Early Detection Contents : Non-Randomly Equivalent Design
}

\author{
Hyun-Seok Jeong, ${ }^{1}$ Oh-Lyong Kim, ${ }^{2}$ Bon-Hoon Koo, ${ }^{3}$ Ki-Hyun Kim, ${ }^{4}$ Gi-Hwan Kim, ${ }^{4}$ Dai-Seg Bai, ${ }^{3}$ Ji-Yean Kim, ${ }^{3}$ \\ Mun-Seon Chang, ${ }^{1, *}$ Hye-Geum Kim ${ }^{3, *}$ \\ Department of Psychology, College of Social Science, Kyungpook National University, Daegu, Korea \\ Department of Neurosurgery, ${ }^{2}$ College of Medicine, Yeungnam University, Daegu, Korea \\ Department of Psychiatry, ${ }^{3}$ College of Medicine, Yeungnam University, Daegu, Korea \\ Corporate Research Institute of Clupea, Inc., ${ }^{4}$ Daegu, Korea
}

Objective : The aim of this study was to verify the equivalence and effectiveness of the tablet-administered Korean Repeatable Battery for the Assessment of Neuropsychological Status (K-RBANS) for the prevention and early detection of dementia.

Methods : Data from 88 psychiatry and neurology patient samples were examined to evaluate the equivalence between tablet and paper administrations of the K-RBANS using a non-randomly equivalent group design. We calculated the prediction scores of the tablet-administered K-RBANS based on demographics and covariate-test scores for focal tests using norm samples and tested format effects. In addition, we compared the receiver operating characteristic curves to confirm the effectiveness of the K-RBANS for preventing and detecting dementia.

Results : In the analysis of raw scores, line orientation showed a significant difference ( $t=-2.94, p<0.001)$, and subtests showed small to large effect sizes (0.04-0.86) between paper- and tablet-administered K-RBANS. To investigate the format effect, we compared the predicted scaled scores of the tablet sample to the scaled scores of the norm sample. Consequently, a small effect size ( $d \leq 0.20)$ was observed in most of the subtests, except word list and story recall, which showed a medium effect size $(\mathrm{d}=0.21)$, while picture naming and subtests of delayed memory showed significant differences in the one-sample t-test. In addition, the area under the curve of the total scale index (TSI) $(0.827 ; 95 \%$ confidence interval, $0.738-0.916)$ was higher than that of the five indices, ranging from 0.688 to 0.820 . The sensitivity and specificity of TSI were $80 \%$ and $76 \%$, respectively.

Conclusion : The overall results of this study suggest that the tablet-administered K-RBANS showed significant equivalence to the norm sample, although some subtests showed format effects, and it may be used as a valid tool for the brief screening of patients with neuropsychological disorders in Korea.

Key Words : Equivalence trial · Neuropsychological test · Research methodology · Korean Repeatable Battery for the Assessment of Neuropsychological Status (K-RBANS) · Dementia.

- Received : June 18, 2021 • Revised : July 21, 2021 •Accepted : July 28, 2021

- Address for reprints : Mun-Seon Chang

Department of Psychology, College of Social Science, Kyungpook National University, 80 Daehak-ro, Buk-gu, Daegu 41566, Korea

Tel : +82-53-950-7161, Fax : +82-53-950-5243, E-mail : moonsun@knu.ac.kr, ORCID : https://orcid.org/0000-0002-0687-1400

\section{Hye-Geum Kim}

Department of Psychiatry, College of Medicine, Yeungnam University, 170 Hyeonchung-ro, Nam-gu, Daegu 42415, Korea

Tel : +82-53-620-0000, Fax : +82-53-620-0000, E-mail : psykhg@yu.ac.kr, ORCID : https://orcid.org/0000-0002-9677-7011

${ }^{*}$ These authors contributed equally to this work.

This is an Open Access article distributed under the terms of the Creative Commons Attribution Non-Commercial License (http://creativecommons.org/licenses/by-nc/4.0) which permits unrestricted non-commercial use, distribution, and reproduction in any medium, provided the original work is properly cited. 


\section{INTRODUCTION}

Currently, the dementia population and management costs are expected to increase along with the increase in the elderly population worldwide. The National Statistical Office reported that the elderly, aged 65 years or older, accounted for 15.7\% of the South Korean population in 2020 and predicted that they would account for $20.3 \%$ in 2025 and $46.5 \%$ in $2067^{23,24)}$. Considering that the elderly population constitutes $9.1 \%$ of the world's population, the increase in the elderly population in Korea is high ${ }^{25}$. Dementia is a typical mental illness that is closely related to the increase in the elderly population; its main symptom is cognitive impairment, including memory degradation and degradation of daily life functions because of changes in personality ${ }^{1)}$. Given that the prevalence of dementia in the Asian population is $5.23 \%{ }^{5}$, the estimated prevalence of dementia above 65 years of age, which reaches $10.53 \%$, and the annual management cost per dementia patient, which is up to KRW 18.51 million, shows the risks for South Korean elder$1 \mathrm{y}^{9,17}$. Aside from dementia, the number of patients is also expected to increase as the elderly population increases; consequently, the need for research and development related to dementia has increased.

In Korea, various support systems, such as community dementia reassurance centers, long-term care services, and medical expenses, are implemented through the national responsibility system for dementia and state affairs. Specifically, the government continues to develop policies that cover the costs of diagnosis and treatment of patients with dementia, such as the application of health insurance for dementia screening costs and introduction of a special calculation system for severe dementia ${ }^{16}$. However, dementia, a degenerative disease, worsens with time, and its risk increases with age; therefore, the social cost must be limited by preventing or alleviating the occurrence and deterioration of dementia. Efficient solutions to dementia-related problems, such as early detection and quick intervention, are required. Moreover, considering the persistent COVID-19 situation, it is expected that the need for non-face-to-face psychological tests will increase rapidly. Nonface-to-face neuropsychological testing is useful in that it can overcome the limitations of time and space required for conducting psychological test. Based on smart devices or online platforms, the psychological characteristics of examinees can be captured using the paradigm of neuropsychological tests without direct contact between examiner and examinee. In particular, the fact that dementia patients, which are one of the main subjects of neuropsychological testing, are often difficult to hang out alone, suggests the need for non-face-to-face neuropsychological testing. In this respect, Korean Repeatable Battery for the Assessment of Neuropsychological Status (KRBANS) as a screening neuropsychological test is not only more sophisticated and systematic than tests such as MiniMental State Examination (MMSE) and clinical dementia rating (CDR) scale, but also has the advantage of conducting test shorter and more efficient than diagnostic tests such as Seoul Neuropsychological Screening Battery (SNSB)-II.

In studies and markets related to dementia, it is expanding around diagnosis in South Korea. In particular, the demand for dementia testing tools that can help predict high-risk dementia groups and prevent dementia is increasing. Screening tests for identifying dementia in Korea include the Korean version of Consortium to Establish a Registry for Alzheimer's Disease $(\mathrm{CERAD}-\mathrm{K})^{18)}$ and SNSB ${ }^{15)}$. These screening tools, used in approximately 40 countries, are comprehensive and inclusive tools that take approximately 30 to 40 minutes for CERAD-K, and approximately 1 hour and 30 minutes to 2 hours for SNSB. However, these screening tests require space and time for trained professionals, resulting in inconveniences and limitations in access and usefulness to prevent dementia. In addition, relatively brief and easy tests developed to identify dementia or mild cognitive impairment (MCI), such as $\mathrm{MMSE}^{10)}$ and $\mathrm{CDR}^{19)}$, are commonly used in clinical settings, but limitations in their accuracy and clinical usefulness have been highlighted ${ }^{11,26)}$. To compensate for these problems, there is a need for a tool that can be used to identify and prevent dementia with accuracy and clinical usefulness while reducing the restrictions on places and efforts needed for testing as much as possible. In this context, the K-RBANS is drawing attention.

The RBANS, which consists of 12 subtests and takes approximately 20 to 30 minutes when conducted by an experienced examiner, is an individual simple test that measures attention, language, visual space composition ability, immediate memory, and delayed memory ${ }^{22)}$. The K-RBANS ${ }^{21}$, standardized for the Korean population, was conducted with the examiner using test papers, targeting individuals between 12 to 60 years or older. Standard scores were collected from 606 healthy adolescents and adults using stratified sampling. In 
particular, the K-RBANS was developed for three purposes. First, as an independent core battery, the K-RBANS was developed to identify and evaluate senile dementia. Second, as a screening battery, the K-RBANS was considered to assess patients who are unable to or unsuitable for standardized assessments, consuming relatively long time and effort. Finally, it was developed in a format that allows repeated evaluations when homomorphism is required to control the practice effect. Although the K-RBANS is designed to be conducted as easily as it is on the bed, there are still inconveniences in the administration and scoring processes. Therefore, this study minimizes inconvenience by materializing the K-RBANS based on tablet PCs and confirms the effectiveness of the tablet-administered K-RBANS using Clinical Data Interchange Standards Consortium ${ }^{3)}$ that enables non-pharmacologic dementia prevention and early detection by examining equivalence with the existing K-RBANS.

\section{MATERIALS AND METHODS}

The study protocol was approved by the Institutional Review Board of Yeungnam University Hospital (IRB No. YUMC 2019-06-054).

\section{Participants}

In this study, we recruited 88 participants from a university hospital. A neuropsychological evaluation was conducted for patients with dementia or patients with suspected dementia who voluntarily agreed to participate in the study, from September to October 2019. The inclusion criteria were a Korean Mini-Mental Status Examination (K-MMSE) score between 10 to 26 and a CDR score between 0.5 to 2 . The exclusion criteria were those patients who 1) required frequent admission or hospitalization, 2) required medical re-access due to rapid deterioration of dementia symptoms, or 3) used internal medicine or had accompanying diseases that may cause cognitive decline.

\section{Materials}

The K-RBANS-update

The K-RBANS ${ }^{21)}$ is an independent and concise examination of attention, language ability, visuospatial/constructional ability, and immediate and delayed memory. This test consists of 12 subtests and is designed to allow skilled examiners to conduct the test for approximately 20 to 30 minutes. Immediate memory consists of list learning and story memory, which measures the examinee's ability to remember immediately after the stimulus is presented. Visuospatial/construction capabilities are indicators consisting of figure copy and line orientation, indicating the ability to copy visual simulations accurately and perceive the spatial relationships of pictures. Language ability consists of picture naming and semantic fluency, reflecting the examinee's ability to verbally recall or name learned objects. Attention refers to the ability to remember and manipulate visually and linguistically presented information in a short period, which is measured by digit span and coding. Delayed memory evaluates the ability of proactive memory, including word list recall, recognition, story, and figure recall. Participants' overall cognition ability could be reflected by the total scale index (TSI), which integrates the above five index scores. In addition, the K-RBANS was designed to target individuals between 12 to 60 years or older, and the norm score was collected and standardized from 606 healthy adolescents and adults using stratified sampling. Moreover, the K-RBANS, developed as a tablet version, was created by Clupea ${ }^{\circledR}$ (Clupea, Daegu, Korea) as an application in accordance with the instructions and procedures of the test paper and manual. Visual stimuli were presented in the tablet according to what was presented in the test paper and stimulus booklet, and instructions and auditory stimuli were presented in a recorded voice. In this process, mental health professionals and two clinical psychologists ensured that the KRBANS stimuli and instructions were properly presented. In this study, we implemented the tablet version K-RBANS on a 10.5 inch screen using Samsung Galaxy Tab S5 (Samsung, Seoul, Korea).

\section{The K-MMSE}

The K-MMSE is an assessment tool developed by Folstein et al. ${ }^{10)}$ for overall cognitive capabilities and cognitive adverse functions. In Korea, Kang et al. ${ }^{14)}$ proposed the K-MMSE, which is widely used in clinical evaluations and research on patients with dementia. The K-MMSE is composed of 30 points : time orientation (5 points), spatial orientation (5 points), memory registration (3 points), attention and calculation ability (5 points), memory recall ( 3 points), language abil- 
ity (8 points), and drawing ability (1 point). Although the MMSE cut-off scores differ depending on the purpose of the study, it is known that scores between 24 and 28 are mainly used to differentiate between patients with MCI and dementia $^{13,14,20)}$. In this study, in terms of practicality in the actual clinical setting, the MMSE score of 26 and the CDR score of 0.5 were selected according to the domestic medical care benefit application standards.

\section{CDR scale}

The CDR scale ${ }^{19)}$ is a semi-structured interview with patients and caregivers. This scale evaluates six cognitive functional areas : memory, orientation, judgment and problemsolving, social activities, household activities and hobbies, and hygiene management, scoring 0 for undamaged; 0.5 , suspected to damage; and 1 to 5 , mild, moderate, severe, and very severe cognitive impairment, respectively. The scores evaluated in all six areas are calculated to "sum of boxes," ranging from 0 to 30 points. Moreover, the overall CDR score is determined using memory as a key factor, whether high or low, among the five cognitive domains, or following the prescribed algorithm.

\section{Procedure}

Patients who voluntarily agreed to participate received a consent form to participate in the study, conducted a simple physical examination, and collected the test results within a year from the date of participation. Participants who did not undergo the K-MMSE or CDR were tested on the day they

Table 1. Administration Procedure by resigned groups

\begin{tabular}{lcc}
\hline & Odd number group Even number group \\
\hline List learning & Paper & Tablet \\
\hline Story memory & Tablet & Paper \\
\hline Figure copy & Paper & Tablet \\
Line orientation & Tablet & Paper \\
\hline Picture naming & Paper & Tablet \\
Semantic fluency & Tablet & Paper \\
Digit span & Paper & Tablet \\
Coding & Tablet & Paper \\
List recall & Paper & Tablet \\
List recognition & Tablet & Paper \\
Story recall & Paper & Tablet \\
Figure recall & Tablet & Paper \\
\hline
\end{tabular}

visited and were registered to participate in the study and assigned to the odd or even groups. Each group was administered with the K-RBANS according to a set order of subtests that alternated between paper and tablet formats. The order of administration of the K-RBANS between the groups is presented in Table 1. This procedure enables the estimation of scaled scores for the tablet-administered K-RBANS using norm samples and compares the format effect between paperand tablet-administered K-RBANS. If a substitution is needed because of a dropout, the number assigned to drop-out participants is given in the order of screening numbers. The test was conducted in a room where noise was blocked, with no distractions, and the K-RBANS was performed in an order by preparing devices for the tester and participants. Compensation for participating in this study was provided to those who completed the test.

\section{Design}

To test equivalence, we used a non-randomly equivalent group design ${ }^{8)}$. A non-randomly equivalent group design can be used if two or more subtests exist to measure one ability construct, and if the norm sample is constructed through appropriate standardization procedures. The performance of the norm sample obtained through the standardization process is used to estimate and compare performance using the tablet version and finally compare the media effect (difference between the existing version and tablet version). Participants in this study were divided into the odd and even groups according to the test subject number (RXXX), and each group was examined in the order shown in Table 1. In this design, the KRBANS norm samples as data that are conducted in a papertype format were utilized to derive a formula for predicting conversion scores to determine the media effect of the KRBANS tablet version. In this study, we compared the differences in the raw scores of the K-RBANS administered on paper and tablets and conducted a regression analysis based on age, number of years of education, gender, and covariate subtests to confirm the media effects of the K-RBANS. Therefore, half of the subtests served as paper-administered covariate tests because the norm and study samples were both administered in paper format, while the other half of the subtests were administered in tablet format to evaluate the format effect. This approach was adopted to maximize the multiple correlations of each subtest in both the halves, which maximizes sta- 
tistical power ${ }^{8}$. In addition, average residuals derived from the difference between observed and predicted scaled scores on tablet-administered subtests imply that the scores from the tablet PC format were higher than predicted in positive value, and vice versa. The effect size was calculated by dividing the average residuals by 3 , which is the standard deviation of the scaled scores in the norm sample.

\section{Statistical analysis}

Prior to the analysis, we excluded cases in which the test results were deemed contaminated because of failure to complete the test or indirect participation in the test. We compared demographic information between the odd and even groups and raw scores of the K-RBANS according to format using an independent t-test (Fisher's exact test for gender distribution by groups). Next, we used multiple regression analysis to estimate scaled scores of the tablet-administered KRBANS based on the effects of sex, age, and education and analyzed format effects using a one-sample t-test of average residuals between observed and predicted scaled scores. In ad- dition, the effect size was calculated to determine the extent of the difference between administration types to confirm format effects rather than judging on dichotomous significance. We also verified the capacity of the tablet-administered KRBANS to screen for cognitive impairment using a receiver operating curve (ROC) analysis. The assessment of statistical significance in all analyses was set at $p<0.05$. The data were analyzed using IBM SPSS Statistics for Windows, version 25.0 (IBM Corp., Armonk, NY, USA) ${ }^{12}$.

\section{RESULTS}

\section{Demographic information}

Of the 88 participants, the average age in the odd and even groups was $67.75 \pm 8.75$ and $68.43 \pm 7.93$, respectively. The odd group consisted of 12 men (27.27\%) and 32 women (72.73\%), and the even group consisted of nine men (20.45\%) and 35 women (79.55\%). The average education years in the odd and even groups were $9.55 \pm 4.82$ and $8.32 \pm 4.36$, respectively. In the

Table 2. Demographic and clinical characteristics of 88 participants

\begin{tabular}{|c|c|c|c|c|}
\hline & \multicolumn{2}{|c|}{ Type of groups } & \multirow{2}{*}{$\begin{array}{l}\text { Total participants } \\
\qquad(\mathrm{n}=88)\end{array}$} & \multirow[b]{2}{*}{$p$-value* } \\
\hline & $\begin{array}{l}\text { Odd number group } \\
\qquad(n=44)\end{array}$ & $\begin{array}{l}\text { Even number group } \\
\qquad(n=44)\end{array}$ & & \\
\hline Age (years) & $67.75 \pm 8.75$ & $68.43 \pm 7.93$ & $68.09 \pm 8.31$ & 0.703 \\
\hline $45-49$ & $2(4.55)$ & $1(2.27)$ & $3(3.41)$ & \\
\hline $50-59$ & $6(13.64)$ & $4(9.09)$ & $10(11.36)$ & \\
\hline $60-69$ & $18(40.91)$ & $21(47.73)$ & $39(44.32)$ & \\
\hline $70-79$ & $14(31.82)$ & $13(29.55)$ & 27 (30.68) & \\
\hline$\geq 80$ & $4(9.09)$ & $5(11.36)$ & $9(10.23)$ & \\
\hline Gender & & & & 0.952 \\
\hline Male & $12(27.27)$ & $9(20.45)$ & $21(23.86)$ & \\
\hline Female & $32(72.73)$ & $35(79.55)$ & $67(76.14)$ & \\
\hline Education & $9.55 \pm 4.82$ & $8.32 \pm 4.36$ & $8.93 \pm 4.61$ & 0.214 \\
\hline$>1$ & $2(4.55)$ & $3(6.82)$ & $5(5.68)$ & \\
\hline $1-6$ & $14(31.82)$ & 15 (34.09) & $29(32.95)$ & \\
\hline $7-9$ & $9(20.45)$ & $12(27.27)$ & $21(23.86)$ & \\
\hline $10-12$ & $10(22.73)$ & $9(20.45)$ & $19(21.59)$ & \\
\hline$\geq 13$ & $9(22.73)$ & $5(11.36)$ & 14 (15.91) & \\
\hline K-MMSE & $24.25(2.67)$ & $24.45(2.35)$ & $24.35(2.50)$ & 0.704 \\
\hline CDR & $0.69(0.38)$ & $0.63(0.22)$ & $0.66(0.31)$ & 0.303 \\
\hline
\end{tabular}

Values are presented as mean \pm standard deviation or number (\%). * $p$-values are derived from independent $t$ test or Fisher's exact test for comparison between groups. K-MMSE : Korean Mini Mental Status Examination, CDR : clinical dementia rating 
Table 3. Comparison between paper and tablet version raw scores of K-RBANS of 88 participants

\begin{tabular}{|c|c|c|c|c|}
\hline & Paper $(n=44)$ & Tablet $(n=44)$ & $\mathrm{t}$ & Cohen's d \\
\hline List learning & $17.80 \pm 5.11$ & $17.07 \pm 6.83$ & 1.01 & 0.12 \\
\hline Story memory & $10.30 \pm 4.00$ & $10.55 \pm 4.29$ & 0.47 & 0.06 \\
\hline Figure copy & $17.59 \pm 3.36$ & $17.32 \pm 3.66$ & 0.56 & 0.08 \\
\hline Line orientation & $12.98 \pm 4.31$ & $9.05 \pm 4.78$ & $-2.94^{*}$ & 0.86 \\
\hline Picture naming & $7.05 \pm 1.94$ & $6.39 \pm 1.93$ & 1.87 & 0.34 \\
\hline Semantic fluency & $12.84 \pm 4.35$ & $11.05 \pm 4.25$ & -1.73 & 0.42 \\
\hline Digit span & $7.30 \pm 2.84$ & $7.18 \pm 2.97$ & 0.13 & 0.04 \\
\hline Coding & $24.30 \pm 11.28$ & $20.43 \pm 11.40$ & -0.94 & 0.34 \\
\hline List recall & $1.75 \pm 1.88$ & $2.48 \pm 2.29$ & 1.42 & 0.35 \\
\hline List recognition & $15.64 \pm 3.50$ & $15.75 \pm 2.62$ & 0.83 & 0.04 \\
\hline Story recall & $4.27 \pm 2.92$ & $4.20 \pm 3.17$ & -0.62 & 0.02 \\
\hline Figure recall & $10.75 \pm 5.59$ & $9.66 \pm 5.70$ & 0.00 & 0.19 \\
\hline
\end{tabular}

Values are presented as mean \pm standard deviation or number. ${ }^{*} p<0.001$. K-RBANS : Korean Repeatable Battery for Assessment of Neuropsychological Status

screening phase, the odd group had an average K-MMSE score of $24.25 \pm 2.67$ points and a CDR score of $0.69 \pm 0.38$, while the even group had a K-MMSE score of $24.45 \pm 2.35$ and a CDR score of $0.63 \pm 0.22$. The demographic information of the study participants in terms of age, gender, education, and cognitive ability did not show significant differences between the odd and even groups. The results are presented in Table 2 .

\section{Comparison of raw scores between original and tablet versions of the K-RBANS}

In Table 3, a comparison of the original K-RBANS conducted in paper and tablet formats showed significant differences in the type of performance in line orientation $(t=-2.94$, $p<0.001)$. In addition, the effect size between the type of KRBANS performance was high in line orientation $(\mathrm{d}=0.86)$. However, it showed moderate effect sizes in picture naming $(\mathrm{d}=0.34)$, semantic fluency $(\mathrm{d}=0.42)$, symbol coding $(\mathrm{d}=0.34)$, and word list recall $(\mathrm{d}=0.35)$ while other subtests showed low effect sizes.

\section{Estimating the scaled scores of the tablet version of the K-RBANS using norm samples}

In this study, we utilized data from a normative group to estimate the scaled scores of the tablet version of the KRBANS. In Table 4, different formulas were estimated using regression analysis based on the following age groups : 40 to
49 years, 50 to 59 years, and 60 years or older. Table 5 shows how well the predictive formulas derived from the norm sample fit the tablet version of the K-RBANS. The correlation coefficients of the norm sample represent the results calculated for each subtest using the prediction formula. All subtests showed correlation coefficients ranging from 0.99 to 1.00 . In the tablet version of the K-RBANS, the correlation coefficient ranged from 0.85 to 1.00 , indicating that the formula calculated from the normative group was also useful in the tablet version.

\section{Media effects of the tablet version of the K-RBANS using estimated scaled scores}

Table 6 shows the analysis of the media effects using observed and predicted scaled scores with tablets, not using scaled scores of the paper-type subtest. The analysis of the difference between the observed and predicted scaled scores (format effects) with a one-sample t-test showed significant differences in the case of zero residual in all subtests of picture naming and in all delayed memory subtests. The predicted scaled score of picture naming showed positive average residuals, while all subtests of delayed memory showed negative average residuals. In addition, the effect size of word list and story recall showed a moderate level in delayed memory, and other subtests showed a low effect size that did not exceed 0.2. 
Table 4. Unstandardized regression weights and constants for the K-RBANS index scores by scaled scores of 218 norm data

\begin{tabular}{|c|c|c|c|}
\hline & $\begin{array}{c}40-49 \text { years } \\
\text { old }(n=75)\end{array}$ & $\begin{array}{c}50-59 \text { years } \\
\text { old }(n=68)\end{array}$ & $\begin{array}{l}\geq 60 \text { years } \\
\text { old }(n=75)\end{array}$ \\
\hline \multicolumn{4}{|l|}{ Immediate memory } \\
\hline $\mathrm{R}$ & 0.997 & 1.000 & 1.000 \\
\hline Constant & 32.169 & 40.303 & 42.979 \\
\hline List learning & 3.360 & 2.962 & 2.852 \\
\hline Story memory & 3.370 & 2.965 & 2.849 \\
\hline \multicolumn{4}{|l|}{ Visospatial capacity } \\
\hline $\mathrm{R}$ & 1.000 & 1.000 & 1.000 \\
\hline Constant & 25.127 & 37.506 & 40.015 \\
\hline Figure copy & 3.716 & 3.131 & 2.999 \\
\hline Line orientation & 3.734 & 3.151 & 2.999 \\
\hline \multicolumn{4}{|l|}{ Language } \\
\hline $\mathrm{R}$ & 1.000 & 1.000 & 1.000 \\
\hline Constant & 37.269 & 36.491 & 41.096 \\
\hline Picture naming & 3.174 & 3.193 & 2.941 \\
\hline Semantic fluency & 3.152 & 3.183 & 2.963 \\
\hline \multicolumn{4}{|l|}{ Attention } \\
\hline $\mathrm{R}$ & 1.000 & 1.000 & 1.000 \\
\hline Constant & 38.097 & 38.127 & 43.242 \\
\hline Digit span & 3.124 & 3.092 & 2.845 \\
\hline Coding & 3.100 & 3.085 & 2.859 \\
\hline \multicolumn{4}{|l|}{ Delayed memory } \\
\hline R & 1.000 & 1.000 & 1.000 \\
\hline Constant & 23.384 & 28.289 & 33.986 \\
\hline List recall & 1.043 & 1.157 & 0.765 \\
\hline List recognition & 3.276 & 3.007 & 2.902 \\
\hline Story recall & 1.246 & 1.156 & 1.099 \\
\hline Figure recall & 2.071 & 1.846 & 1.810 \\
\hline
\end{tabular}

Statistical significances of all fitness-of-model and all subtest are $p<0.001$. K-RBANS : Korean Repeatable Battery for Assessment of Neuropsychological Status

\section{ROC analysis results}

To verify the capacity of the tablet version of the K-RBANS to detect performance impairment in patients with dementia and MCI, we performed ROC analysis using estimated scaled scores in paper- and tablet-administered K-RBANS. The area under curve (AUC) of the TSI was the highest at 0.827 , with a cut-off point of 67.5. In the other five indices, the AUC was shown to be statistically significant, ranging from 688 to 820 . In addition, the sensitivity of the TSI was $80 \%$, and the speci-
Table 5. Applicability of prediction equations derived from scaled scores of norm data to the K-RBANS

\begin{tabular}{lcc}
\hline \multirow{2}{*}{ Subtest } & \multicolumn{2}{c}{$\begin{array}{c}\text { Correlation of predicted scaled score } \\
\text { with actual score }\end{array}$} \\
\cline { 2 - 3 } & $\begin{array}{c}\text { Equivalence sample } \\
(\mathbf{n}=\mathbf{4 4 )}\end{array}$ & $\begin{array}{c}\text { Norm sample } \\
(\mathbf{n}=\mathbf{2 1 8})\end{array}$ \\
\hline List learning & 1.00 & 1.00 \\
Story memory & 1.00 & 1.00 \\
\hline Figure copy & 0.99 & 1.00 \\
Line orientation & 1.00 & 1.00 \\
Picture naming & 0.99 & 1.00 \\
Semantic fluency & 1.00 & 1.00 \\
\hline Digit span & 0.99 & 1.00 \\
Coding & 1.00 & 1.00 \\
List recall & 0.85 & 0.99 \\
List recognition & 0.99 & 1.00 \\
Story recall & 0.84 & 1.00 \\
Figure recall & 0.98 & 1.00 \\
\hline
\end{tabular}

Tablet version samples. Statistical significances of all subtest are $p<0.001$. K-RBANS : Korean Repeatable Battery for Assessment of Neuropsychological Status

ficity was $76 \%$. The results are listed in Table 7.

\section{DISCUSSION}

Recently, there has been a movement to establish a psychological examination paradigm using smart devices and PCs. Q-interactive is an iPad-based examination system that allows clinical evaluations, such as the Wechsler Intelligence Scale for Children-fifth edition, Wechsler Individual Achievement Test-third edition, and Clinical Evaluation of Language Fundamentals-fifth edition, to be conducted, scored, and report$\mathrm{ed}^{27)}$. In Korea, CERAD-K manufactures and distributes applications that can be used in smart devices. However, despite having technology that can implement the neuropsychological examination paradigm in smart devices in Korea, there is not enough verification of its effectiveness. For example, in the case of CERAD-K, the equivalence and effectiveness of applications for smart devices have not been verified, which limits the interpretation of the test results. In this study, we aimed to confirm whether the K-RBANS performance differs in paper and tablet administrations and determine whether the tablet- 
Table 6. K-RBANS format effects of 44 participants using scaled scores

\begin{tabular}{lccc}
\hline Subtest & Residual & t & Effect size \\
\hline List learning & $0.017 \pm 0.102$ & 1.107 & 0.006 \\
Story memory & $0.006 \pm 0.102$ & 0.375 & 0.002 \\
\hline Figure copy & $0.064 \pm 0.439$ & 0.973 & 0.021 \\
Line orientation & $-0.007 \pm 0.053$ & -0.874 & -0.002 \\
Picture naming & $0.038 \pm 0.103$ & $2.447^{*}$ & 0.013 \\
Semantic fluency & $0.018 \pm 0.095$ & 1.254 & 0.006 \\
Digit span & $0.040 \pm 0.350$ & 0.761 & 0.013 \\
Coding & $-0.014 \pm 0.100$ & -0.951 & -0.005 \\
List recall & $-0.841 \pm 2.194$ & $-2.544^{*}$ & -0.280 \\
List recognition & $-0.220 \pm 0.546$ & $-2.670^{*}$ & -0.073 \\
Story recall & $-0.657 \pm 1.814$ & $-2.405^{*}$ & -0.219 \\
Figure recall & $-0.352 \pm 0.873$ & $-2.675^{*}$ & -0.117 \\
\hline
\end{tabular}

Values are presented as mean \pm standard deviation or number (\%). ${ }^{*} p<0.05$. K-RBANS : Korean Repeatable Battery for Assessment of Neuropsychological Status, Residual : differences between observed and predicted scores, $t$ : $t$ value derived from one-sample $t$ test, Effect size : mean residuals / 3 (standard deviation of scaled scores of K-RBANS)

Table 7. Screening ability on the cut-off scaled score of K-RBANS using K-MMSE $=26$ and CDR=0.5 among 88 participants

\begin{tabular}{lccccc}
\hline & Cutoff score & AUC (95\% Cl) & Sensitivity (\%) & Specificity (\%) & $p$-value \\
\hline Immediate memory & $\leq 78.0$ & $0.706(0.575-0.836)$ & 66.18 & 65.00 & 0.005 \\
Visuospatial capacity & $\leq 89.5$ & $0.765(0.657-0.874)$ & 66.18 & 80.00 & $<0.001$ \\
Language & $\leq 77.0$ & $0.800(0.709-0.891)$ & 69.12 & 65.00 & 72.06 \\
Attention & $\leq 76.5$ & $0.688(0.557-0.819)$ & 60.00 & 72.06 & 0.011 \\
Delayed memory & $\leq 72.5$ & $0.820(0.721-0.919)$ & 75.00 & 801 & $<0.001$ \\
Total scale & $\leq 67.5$ & $0.827(0.738-0.916)$ & 80.00 & $<.47$ & $<0.001$ \\
\hline
\end{tabular}

K-RBANS : Korean Repeatable Battery for Assessment of Neuropsychological Status, K-MMSE : Korean Mini-Mental State Examination, CDR : clinical dementia rating, $\mathrm{Cl}$ : confidence interval, $\mathrm{AUC}$ : area under the curve

administered K-RBANS can detect and identify patients with dementia and MCI.

We compared paper- and tablet-administered K-RBANS performances using raw scores. In the results of the one-sample t-test, line orientation showed a significantly low score in the tablet-administered K-RBANS. Moreover, line orientation showed a large effect size; picture naming, semantic fluency, coding, and word list recall showed medium effect sizes; and other subtests showed low effect sizes. Given that raw scores of the tablet-administered K-RBANS have limitations using the norm sample of paper-administered K-RBANS because it would be easily affected by age, education, and gender, raw scores would be more sensitive to the format effect. However, it needs to be refined carefully so that the equivalence of raw scores can reduce potential threats to the adaptation of psychological tests ${ }^{6}$.

Next, we estimated scaled scores of the tablet-administered K-RBANS to verify format effects using demographic variables and covariate subtests with the norm sample. In this process, we produced prediction equations derived from scaled scores of norm data and estimated scaled scores in the tablet-administered K-RBANS to confirm the format effect. Consequently, in a one-sample t-test, picture naming, word list recall, list recognition, story, and figure recall showed significant differences but low effect sizes, and word list and story recall showed low effect sizes. This result means that some subtests differ when the performance of the tablet-administered K-RBANS is converted into a scaled score of the norm 
sample. Specifically, with respect to the predicted scaled scores with high correlations to the norm sample, observed scores that are smaller than predicted scores in the tablet-administered K-RBANS suggest that participants' performance in word list and story recall partially meet the expected level. However, given that some psychological tests that were administered digitally showed format effects at this level ${ }^{2,4,7,8)}$, the results that showed low format effects of the tablet-administered K-RBANS appeared to be acceptable. In particular, the average residuals of delayed memory showed a negative value, indicating that the predicted scaled scores were higher than the observed scaled scores. This result suggests that the scaled score is a proper measurement for the soothing effects of demographic variables such as age and that future studies need to elaborate this further, because actual performance was lower than predicted in delayed memory. In addition, in the signal detection analysis for the tablet-administered K-RBANS, all indices, including the TSI, were found to be significant, and the sensitivity and specificity of the TSI were the most suitable for detecting cognitive impairment.

However, this study has several limitations. First, there was no control for matching gender, age, and education between the groups. Although we used scaled scores of the tablet-administered K-RBANS based on age and education, it is known that neuropsychological assessment could be affected by demographic variables. We had a limitations in comparing performance between paper- and tablet-administered K-RBANS based on age, gender, and education directly because of the number of participants and their distribution. Second, we did not measure all the performance on the tablet-administered K-RBANS; therefore, it was not possible to compare test results between paper and tablet administrations directly within participants' capacity. If possible, future research should confirm the test-retest reliability.

Nonetheless, the study results suggest that the K-RBANS has adherent reliability and validity for neuropsychological assessment tools in paper administration and can also be used in tablet administration for screening cognitive decline or impairment because of dementia. This could make users relatively free of limitations related to testing time and place, by administering neuropsychological tests in electronic devices such as tablet PCs. The conversion of K-RBANS to a smart device can contribute to early detection of subjects with mild neurocognitive impairment or dementia. It is thought to be useful in cases where it is difficult to move alone to receive a neuropsychological test due to reduced function or inconvenience in movement. Furthermore, the conversion of neuropsychological testing to smart devices can be a stepping stone to overcome the temporal and spatial limitations of traditional neuropsychological testing when linked with an online platform.

\section{CONCLUSION}

In this study, we confirmed equivalence of tablet-administrated K-RBANS using non-randomly equivalence group design. First, we found that raw scores had some limits for scaled scores using norm samples that was collected by standardization of paper-administrated K-RBANS. Second, in order to estimate the equivalence of tablet-administrated K-RBANS performance correcting effects of demographic characteristics, the formula were yielded from the norm sample. Using this formula, we compared observed and predicted scores, and verified equivalence of K-RBANS enough to use norm sample. Finally, tablet-administrated K-RBANS, as a screening tool, showed good sensitivity and specificity.

\section{CONFLICTS OF INTEREST}

No potential conflict of interest relevant to this article was reported.

\section{INFORMED CONSENT}

Informed consent was obtained from all individual participants included in this study.

\section{AUTHOR CONTRIBUTIONS}

\author{
Conceptualization : HSJ, KHK, BHK, MSC, HGK \\ Data curation : HSJ, DSB, JYK, KHK \\ Formal analysis : HSJ, DSB, GHK \\ Funding acquisition : DSB, BHK, KHK, GHK \\ Methodology : HSJ, OLK, MSC, HGK
}


Project administration : JYK, DSB, BHK, KHK

Visualization : HSJ, KHK, GHK, JYK

Writing - original draft : HSJ, DSB, JYK

Writing - review \& editing : KHK, BHK, OLK, MSC, GHK

\section{ORCID}

Hyun-Seok Jeong https://orcid.org/0000-0002-1613-5001

Oh-Lyong Kim https://orcid.org/0000-0001-9946-2085

Bon-Hoon Koo https:/orcid.org/0000-0001-9633-3835

Ki-Hyun Kim https:/orcid.org/0000-0001-9717-6069

Gi-Hwan Kim https://orcid.org/0000-0001-5711-9350

Dai-Seg Bai https:/orcid.org/0000-0002-6907-6201

Ji-Yean Kim https://orcid.org/0000-0002-4469-1582

Mun-Seon Chang https://orcid.org/0000-0002-0687-1400

Hye-Geum Kim https://orcid.org/0000-0002-9677-7011

\section{- Acknowledgements}

We would like to thank Editage (www.editage.co.kr) for English language editing.

\section{References}

1. American Psychiatric Association : Diagnostic and statistical manual of mental disorders (DSM-5®). Arlington : American Psychiatric Publishing, 2013

2. Anowar M, McGrath C, Saub R : Measurement equivalence of "touchscreen" versus "paper-based" assessments of OHRQoL: a randomized crossover trial. Clin Cosmet Investig Dent 12 : 199-204, 2020

3. Babre DK : Clinical data interchange standards consortium: a bridge to overcome data standardisation. Perspect Clin Res 4 : 115-116, 2013

4. Bailey SKT, Neigel AR, Dhanani LY, Sims VK : Establishing measurement equivalence across computer- and paper-based tests of spatial cognition. Hum Factors 60 : 340-350, 2018

5. Cao Q, Tan CC, Xu W, Hu H, Cao XP, Dong Q, et al. : The prevalence of dementia: a systematic review and meta-analysis. J Alzheimers Dis 73 : 1157-1166, 2020

6. Daniel $M$, Wahlstrom $D$ : Raw-score equivalence of computer-assisted and paper versions of WISC-V. Psychol Serv 16 : 213-220, 2019

7. Daniel $\mathrm{MH}$ : Equivalence of Q-interactive and paper administrations of cognitive tasks: Selected NEPSY®-II and CMS subtests (Q-interactive technical report 4). Bloomington : Pearson, 2013

8. Daniel MH. Equivalence of Q-interactive administered cognitive tasks: WAIS $®$-IV. Bloomington : Pearson Education, Inc, 2012
9. National Institute of Dementia : The status of dementia prevalence. Available at : https://www.nid.or.kr/info/ub_2020.aspx?no=89680

10. Folstein MF, Folstein SE, McHugh PR : "Mini-mental state". A practical method for grading the cognitive state of patients for the clinician. J Psychiatr Res 12 : 189-198, 1975

11. Galasko DR, Gould RL, Abramson IS, Salmon DP : Measuring cognitive change in a cohort of patients with Alzheimer's disease. Stat Med 19 : $1421-1432,2000$

12. IBM Corp. : IBM SPSS statistics for windows version 25. Armonk : IBM Corp., 2018

13. Kang $Y:$ A normative study of the Korean Mini-Mental State Examination (K-MMSE) in the elderly. Korean J Psychology 25 : 1-12, 2006

14. Kang $Y, N a D L$, Hahn S : A validity study on the Korean Mini-Mental State Examination (K-MMSE) in dementia patients. J Korean Neurol Assoc $15:$ 300-308, 1997

15. Kang YO JS, Na DL : Seoul Neuropsychological Screening Battery (SNSB-II), 2nd ed. Seoul : Human Brain Research \& Consulting Co, 2012

16. Lee DW : What is needed for the success of national responsibility for dementia. J Korean Med Assoc 60 : 618-621, 2017

17. Lee J, Kang M, Lee O, Lee H, Kwak M, Yu W, et al. : Korean dementia observatory 2020. Seoul : National Institute of Dementia, 2021

18. Lee JH, Lee KU, Lee DY, Kim KW, Jhoo JH, Kim JH, et al. : Development of the Korean version of the Consortium to Establish a Registry for Alzheimer's Disease Assessment Packet (CERAD-K): clinical and neuropsychological assessment batteries. J Gerontol B Psychol Sci Soc Sci 57 : P47-P53, 2002

19. Morris JC : The Clinical Dementia Rating (CDR): current version and scoring rules. Neurology $43:$ 2412-2414, 1993

20. Oh E, Kang Y, Shin JH, Yeon BK : A validity study of K-MMSE as a screening test for dementia: comparison against a comprehensive neuropsychological evaluation. Dement Neurocognitive Disord 9 : $8-12,2010$

21. Park JO, Koo BH, Kim JY, Bai DS, Chang MS, Kim OL : The Korean repeatable battery for the assessment of neuropsychological statusupdate : psychiatric and neurosurgery patient sample validity. J Korean Neurosurg Soc 64 : 125-135, 2021

22. Randolph C : RBANS update manual. San Antonio : The Psychological Corporation, 2012

23. Statistics Korea : 2020 Senior Statistics. Daejeon : Statistics Korea, 2020

24. Statistics Korea : Future Population Special Estimation: 2017 2067 year. Daejeon : Statistics Korea, 2019

25. UN DESA. World population prospects: the 2019 revision: volume I: comprehensive tables. New York : United Nations, 2019

26. Wada-Isoe K, Kikuchi T, Umeda-Kameyama Y, Mori T, Akishita M, Nakamura $Y$, et al. : Global clinical dementia rating score of 0.5 may not be an accurate criterion to identify individuals with mild cognitive impairment. J Alzheimers Dis Rep 3 : 233-239, 2019

27. Wahlstrom D, Daniel M, Weiss LG, Prifitera A : Chapter 11—Digital Assessment with Q-interactive in Weiss LG, Saklofske DH, Holdnack JA, Prifitera A (eds) : WISC-V Assessment and Interpretation. Cambridge : Academic Press, 2016, pp347-372 